\title{
CAMERA CALIBRATION USING A 3D TRANSFORMATION MATHEMATICAL MODEL
}

\author{
Abdouslam M. Bashir Author \\ Department of Mechatronics \\ Higher Institute of Industrial Technology \\ Tripoli-Libya
}

\begin{abstract}
This paper presents a mathematical model for video sequence calibration when a standard camera is mounted on a five degree-of-freedom fixture. A 3D transformation was used in order to establish the absolute position and orientation of the camera. In this approach, it has been assumed that the bottom left hand corner of the left wall is the origin $(0,0,0)$. This origin was selected because WTK coordinate system adopts a right-hand coordinate frame as stated in Sense8 Inc. literature (1999). The $Y$ coordinate has been inverted to match with that of the static camera. A respectable registration between the real and virtual scenes was achieved in an augmented reality scene following the calibration. The transformation of the camcorder coordinates was used to set up the virtual sense, although minor dynamic errors were found.
\end{abstract}

Keywords - calibration, mathematical transformation.

\section{INTRODUCTION}

It is clear in $3 \mathrm{D}$ computer vision, camera calibration is a necessary step to extract metric

information from 2D images. Extensive studies have been carried away over the years in computer vision and photogrammetry with various techniques being proposed. In this chapter, we review the techniques proposed in the literature include those using 3D apparatus (two or three planes orthogonal to each other, or a plane undergoing a pure translation, etc.), 2D objects (planar patterns undergoing unknown motions), 1D objects (wand with dots) and unknown scene points in the environment (self-calibration).

Tsai created a popular technique for three-dimensional (3D) camera calibration for machine vision metrology using offthe-shelf TV cameras and lenses is described by Roge (1987).
The two-stage technique has advantage in terms of accuracy, speed, and versatility over existing state of the art. Dias (2003) argued that results from the technique though showed that the two-stage calibration could be done in real time with it could be achieved with slight modification.

Alvarez,et et al.(2012) designed several mathematical models for video sequence calibration as cameras are mounted on a tripod. The calibration, in this method, was based on the geometry of the tripod and a primitive tracking procedure which uses lines and circles as primitive. A Classification and Regression Tree (CART) was used for the extraction of primitive information. The quality of the camera calibration procedure was examined by inserting virtual elements in the video sequence.

An interesting method made by Vasconcelos et al. (2012) which a minimal algorithm for fully calibrating a camera from 11 independent pairwise point correspondences with two other calibrated cameras.

The proposed algorithm could be used to insert or re-calibrate a new camera into an existing network, without having to interrupt operation. However, Bazargani (2015) provided an extensive review of some techniques proposed in the literature on camera calibration techniques including involving camera pose estimation and distance estimation.

\section{CAMERA CALIBRATION}

The position and orientation of the camera has to be known in order to have both real and virtual worlds aligned. A mounting fixture was made to constrain the five degree-offreedom camera body (X, Y, Z, pitch, yaw), as shown in figures 1 and 2 . 


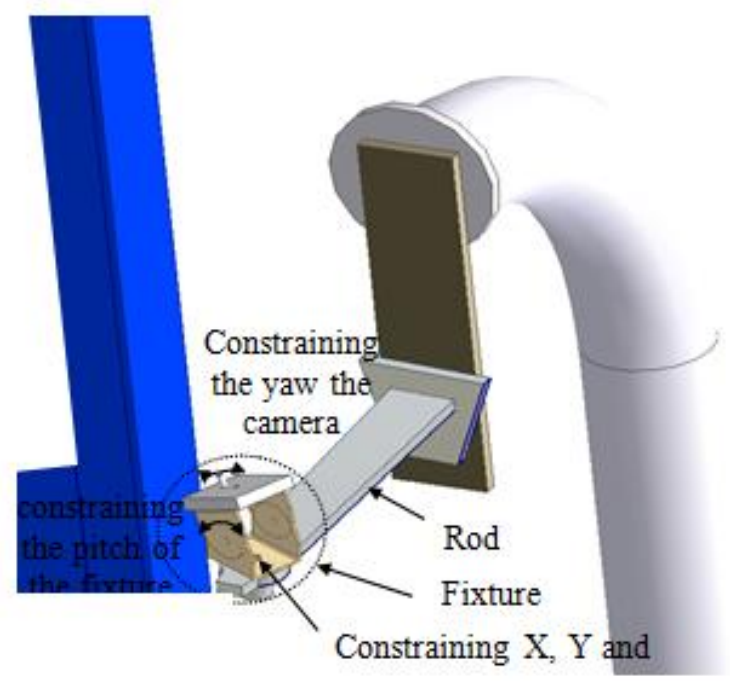

Fig. 1. Camera alignment with the mounting component

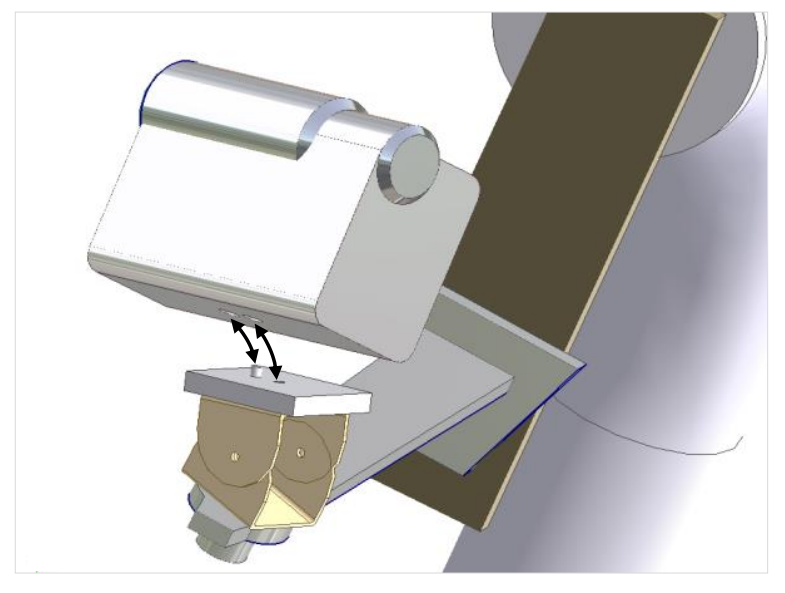

Fig. 2. Attaching the camera to the fixture

To establish the absolute position and orientation of the camera, a 3D transformation was used. In this method it has been assumed that the bottom left hand corner of the left wall is the origin $(0,0,0)$. This origin was selected because Word Tool Kit coordinate system adopts a right-hand coordinate frame as stated in Sense8 Inc. (1999). The Y coordinate has been inverted to match with that of the static camera.

WTK supports $4 \times 4$ homogeneous transformation matrices, which represent translation, scaling and rotation in 3D space. Points are given a fourth coordinate $\mathrm{W}(\mathrm{W} \neq 0)$, whereby each point in 3-space is represented by a line and expressed as (X, $\mathrm{Y}, \mathrm{Z}, \mathrm{W})$ or $(\mathrm{X} / \mathrm{W}, \mathrm{Y} / \mathrm{W}, \mathrm{Z} / \mathrm{W}, 1)$. The transformation of a given point in $3 \mathrm{D}$ space as a subspace of $4 \mathrm{D}$ space, which is given by dividing $\mathrm{X}, \mathrm{Y}$ and $\mathrm{Z}$ by $\mathrm{W}$ (where $\mathrm{W}=1$ ), is known as homogenisation as described Foley et al. (1994).

Translation $T\left(d_{x}, d_{y}, d_{z}\right)$ of a point in 3D space is carried out using the $4 x 4$ matrix

$\mathrm{T}\left(\mathrm{d}_{x}, \mathrm{~d}_{y}, \mathrm{~d}_{z}\right)=\left[\begin{array}{cccc}1 & 0 & 0 & d x \\ 0 & 1 & 0 & d y \\ 0 & 0 & 1 & d z \\ 0 & 0 & 0 & 1\end{array}\right]$

Scaling $\mathrm{S}\left(\mathrm{s}_{\mathrm{x}}, \mathrm{s}_{\mathrm{y}}, \mathrm{s}_{\mathrm{z}}\right)$ of a point can be expressed similarly as

$\mathrm{S}\left(\mathrm{s}_{\mathrm{x}}, \mathrm{s}_{\mathrm{y}}, \mathrm{s}_{\mathrm{z}}\right)=\left[\begin{array}{cccc}s x & 0 & 0 & 0 \\ 0 & s y & 0 & 0 \\ 0 & 0 & s z & 0 \\ 0 & 0 & 0 & 1\end{array}\right]$.

The rotation in 3D space can be performed using the following 3 equations:

$\mathrm{R}_{\mathrm{x}}(\theta)=\left[\begin{array}{cccc}1 & 0 & 0 & 0 \\ 0 & \cos \theta & -\sin \theta & 0 \\ 0 & \sin \theta & \cos \theta & 0 \\ 0 & 0 & 0 & 1\end{array}\right]$,

$\mathrm{R}_{\mathrm{y}}(\theta)=\left[\begin{array}{cccc}\cos \theta & 0 & \sin \theta & 0 \\ 0 & 1 & 0 & 0 \\ -\sin \theta & 0 & \cos \theta & 0 \\ 0 & 0 & 0 & 1\end{array}\right]$,

and

$\mathrm{R}_{\mathrm{z}}(\theta)=\left[\begin{array}{cccc}\cos \theta & -\sin \theta & 0 & 0 \\ \sin \theta & \cos \theta & 0 & 0 \\ 0 & 0 & 1 & 0 \\ 0 & 0 & 0 & 1\end{array}\right]$

where $\theta$ the angle of rotation.

The dimensions of the camcorder are $(73 \times 100 \times 146 \mathrm{~mm})$ as shown in figure 3 . 


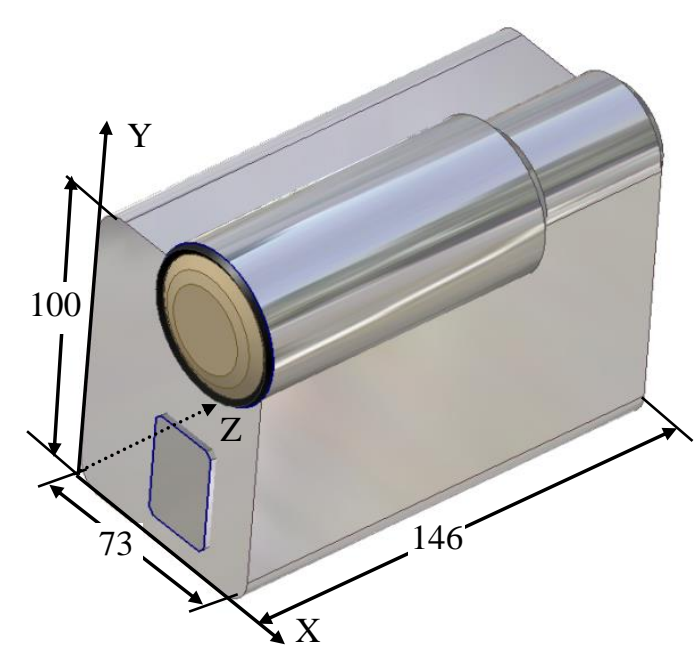

Fig. 3. The camcorder coorditate system and physical dimintions

The camera location, in terms of the 3 primitive transformations ( $\mathrm{T}, \mathrm{Rx}$, and $\mathrm{Ry}$ ) are required to be composed, which involves the 3 points $\left(P_{1}, P_{2}\right.$ and $\left.P_{3}\right)$ being defined as the camera's extents (see figure 4 ). Since the frame and the fixure are co-incident on the same angle around the $\mathrm{Z}$ axis, $\mathrm{Rz}$ need be considered.

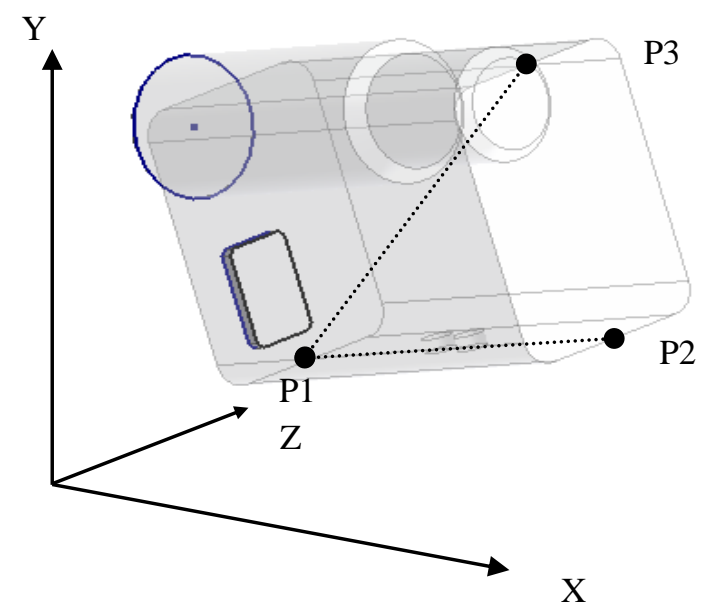

Fig. 4. Defining points on the camcorder body

The procedure was performed in three steps illustrated by Foley et al. (1994), as follows:

$$
\text { Step 1. Translate } P_{1} \text { to the origin. }
$$

Using the translation

$$
T\left(-x_{1},-y_{1},-z_{1}\right)=\left[\begin{array}{cccc}
1 & 0 & 0 & -x_{1} \\
0 & 1 & 0 & -y_{1} \\
0 & 0 & 1 & -z_{1} \\
0 & 0 & 0 & 1
\end{array}\right]
$$

Applying $T$ to $P_{1}, P_{2}$ and $P_{3}$ gives

$$
P_{1}^{\prime}=T\left(-x_{1},-y_{1},-z_{1}\right) \cdot P_{1}=\left[\begin{array}{l}
0 \\
0 \\
0 \\
1
\end{array}\right],
$$

$$
P_{2}^{\prime}=T\left(-x_{1},-y_{1},-z_{1}\right) \cdot P_{2}=\left[\begin{array}{c}
x_{2}-x_{1} \\
y_{2}-y_{1} \\
z_{2}-z_{1} \\
1
\end{array}\right] \text {, }
$$

$P_{3}^{\prime}=T\left(-x_{1},-y_{1},-z_{1}\right) \cdot P_{3}=\left[\begin{array}{c}x_{3}-x_{1} \\ y_{3}-y_{1} \\ z_{3}-z_{1} \\ 1\end{array}\right]$,

Step 2: Rotate about the Y-axis: (figure 5 shows P1P2 after step 1), along with the projection of P1P2 onto the XZ plane. The angle of rotation is $-(90-\theta)=\theta-90$. Then

$$
\begin{aligned}
& \cos (\theta-90)=\sin \theta=\frac{z_{2}^{\prime}}{D_{1}}=\frac{z_{2}-z_{1}}{z_{1}} . \\
& \sin (\theta-90)=-\cos \theta=-\frac{x_{2}^{\prime}}{D_{1}}=-\frac{x_{2}-x_{1}}{D_{1}} . \\
& D_{1}=\sqrt{\left(z_{2}^{\prime}\right)^{2}+\left(x_{2}^{\prime}\right)^{2}}=\sqrt{\left(z_{2}-z_{1}\right)^{2}+\left(x_{2}-x_{1}\right)^{2}} .
\end{aligned}
$$

Where the values are substituted into equation 4

$P_{2}^{\prime \prime}=R_{y}(\theta-90) \cdot P_{2}^{\prime}$. 


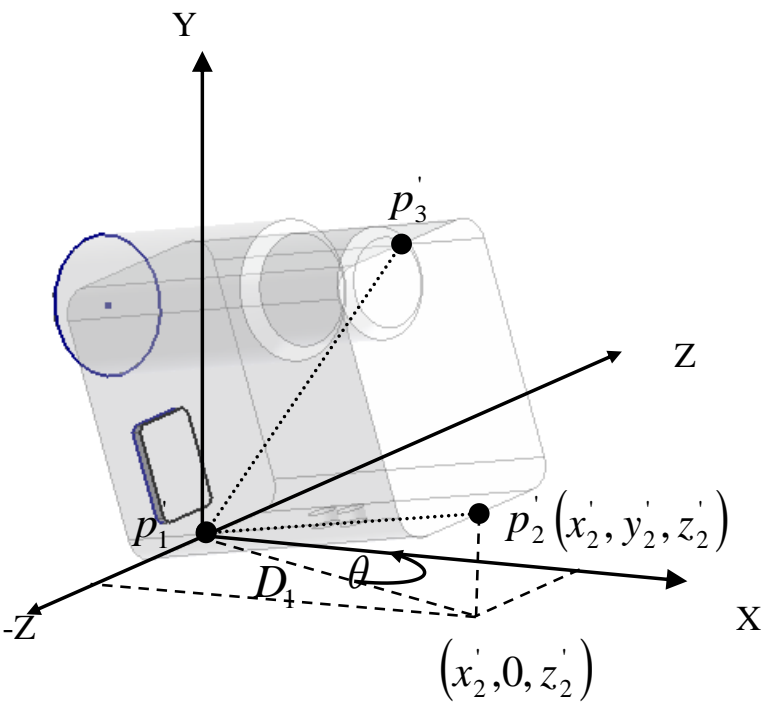

Figure 5 Camcorder translation after translating P1 to origin

Step 3: Rotate about the $\mathrm{X}$-axis. (figure 6 shows $P_{1} P_{2}$ after step 2). The angle of rotation is $\phi$, for which $\cos \phi=\frac{z_{2}^{\prime \prime}}{D_{2}}, \sin \phi=\frac{x_{2}^{\prime \prime}}{D_{2}}$.

Where $D_{2}=\left|P_{1}^{\prime \prime} P_{2}^{\prime \prime}\right|$, The length of $P_{1}^{\prime \prime} P_{2}^{\prime \prime}$. The length of line $P_{1}^{\prime \prime} P_{2}^{\prime \prime}$ is the same as the length of line $P_{1} P_{2}$, since the rotation and translation transformations preserve length, so $D_{2}=\left|\begin{array}{ll}P_{1}^{\prime \prime} & P_{2}^{\prime \prime}\end{array}\right|=\left|\begin{array}{ll}P_{1} & P_{2}\end{array}\right|=\sqrt{\left(x_{2}-x_{1}\right)^{2}+\left(y_{2}-y_{1}\right)^{2}+\left(z_{2}-z_{1}\right)^{2}} 15$

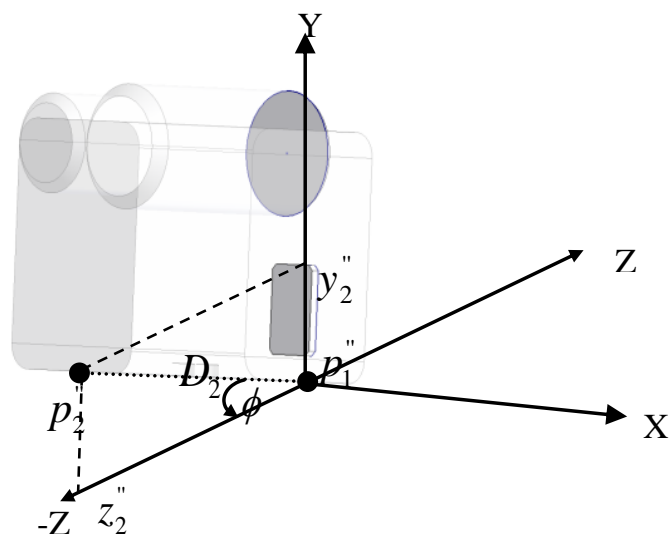

The result of rotation in step 3 is

$$
P_{2}^{\prime \prime \prime}=R_{x}(\phi) \cdot P_{2}^{\prime \prime}=R_{x}(\phi) \cdot R_{y}(\theta-90) \cdot P_{2}^{\prime}=R_{x}(\phi) \cdot R_{y}(\theta-90) \cdot T \cdot P_{2} \cdot 16
$$

Figure 6 Camcorder rotation about $\mathrm{x}$-axis
The following example is an implementation of the 3 primitive transformations on the actual static camcorder (all values are based on AutoCAD 2000 outputs, and are in mm).

Step 1.

$$
\begin{aligned}
& P_{1}=(1253.85,394.39,-279.44) . \\
& P_{2}=(1359.97,424.74,-361.08) . \\
& P_{3}=(1340.13,522,56,-375) .
\end{aligned}
$$

Applying $T$ to $P_{1}, P_{2}$ and $P_{3}$ gives

$$
P_{1}^{\prime}=T\left(-x_{1},-y_{1},-z_{1}\right) \cdot P_{1}=\left[\begin{array}{cccc}
1 & 0 & 0 & -1235.85 \\
0 & 1 & 0 & -394.39 \\
0 & 0 & 1 & 279.44 \\
0 & 0 & 0 & 1
\end{array}\right] \cdot\left[\begin{array}{c}
1235.85 \\
394.39 \\
-279.44 \\
1
\end{array}\right]=\left[\begin{array}{l}
0 \\
0 \\
0 \\
1
\end{array}\right] \text {, }
$$

$$
P_{2}^{\prime}=T\left(-x_{1},-y_{1},-z_{1}\right) \cdot P_{2}=
$$

$\left[\begin{array}{cccc}1 & 0 & 0 & -1235.85 \\ 0 & 1 & 0 & -394.39 \\ 0 & 0 & 1 & 279.44 \\ 0 & 0 & 0 & 1\end{array}\right] \cdot\left[\begin{array}{c}1359.97 \\ 424.74 \\ -361.06 \\ 1\end{array}\right]=\left[\begin{array}{c}106.12 \\ 30.35 \\ -81.64 \\ 1\end{array}\right], 5.21$

$P_{3}^{\prime}=T\left(-x_{1},-y_{1},-z_{1}\right) \cdot P_{3}=$

$$
\left[\begin{array}{cccc}
1 & 0 & 0 & -1235.85 \\
0 & 1 & 0 & -394.39 \\
0 & 0 & 1 & 279.44 \\
0 & 0 & 0 & 1
\end{array}\right] \cdot\left[\begin{array}{c}
1340.13 \\
522.56 \\
-375 \\
1
\end{array}\right]=\left[\begin{array}{c}
86.28 \\
128.17 \\
-95.56 \\
1
\end{array}\right],
$$

Step 2: rotate about the $\mathrm{Y}$-axis is given as

$$
D_{1}=\sqrt{(81.64)^{2}+(106.12)^{2}}=133.89 \text {. }
$$

So,

$$
\sin \theta=\frac{-81.64}{144.89}=-0.60,-\cos \theta=-\frac{106.12}{133.89}=-0.79 .
$$

Thus,

$$
\begin{aligned}
& \theta=-37.57 . \\
& P_{2}^{\prime \prime}=R_{y}(\theta-90) \cdot P_{2}^{\prime} .
\end{aligned}
$$




$$
\begin{gathered}
R_{y}(\theta-90)=\left[\begin{array}{cccc}
\cos (\theta-90) & 0 & \sin (\theta-90) & 0 \\
0 & 1 & 0 & 0 \\
-\sin (\theta-90) & 0 & \cos (\theta-90) & 0 \\
0 & 0 & 0 & 1
\end{array}\right]=\left[\begin{array}{cccc}
\sin \theta & 0 & -\cos \theta & 0 \\
0 & 1 & 0 & 0 \\
\cos \theta & 0 & \sin \theta & 0 \\
0 & 0 & 0 & 1
\end{array}\right] \\
=\left[\begin{array}{cccc}
-0.60 & 0 & -0.79 & 0 \\
0 & 1 & 0 & 0 \\
0.79 & 0 & -0.60 & 0 \\
0 & 0 & 0 & 1
\end{array}\right] .
\end{gathered}
$$$$
27
$$

So,

$$
P_{2}^{\prime \prime}=\left[\begin{array}{cccc}
-0.60 & 0 & -0.79 & 0 \\
0 & 1 & 0 & 0 \\
0.79 & 0 & -0.60 & 0 \\
0 & 0 & 0 & 1
\end{array}\right] \cdot\left[\begin{array}{c}
106.12 \\
30.35 \\
-81.64 \\
1
\end{array}\right]=\left[\begin{array}{c}
0 \\
30.35 \\
133.89 \\
1
\end{array}\right]
$$$$
\text { Or }\left[\begin{array}{llll}
0 & y_{2}-y_{1} & D & 1
\end{array}\right]^{T}
$$

As expected, the $\mathrm{X}$ component of $P_{2}^{\prime \prime}$ is zero, and the $\mathrm{Z}$ component is the length D1.

Step 3: Rotate about the $\mathrm{X}$-axis.

$$
D_{2}=\sqrt{(106.12)^{2}+(30.35)^{2}+(-81.64)^{2}}=137.28 \text {. }
$$

The result of rotation in step 3 is

$$
P_{2}^{\prime \prime}=\left[\begin{array}{llll}
x_{2}^{\prime \prime} & y_{2}^{\prime \prime} & z_{2}^{\prime \prime} & 1
\end{array}\right]^{T}=P_{2}^{\prime \prime}=\left[\begin{array}{llll}
0 & 30.35 & 133.89 & 1
\end{array}\right]^{T} . .30
$$$$
\cos \phi=\frac{133.89}{137.28}=0.79 \text {. }
$$

$\sin \phi=\frac{30.35}{137.28}=0.22$.

$$
\text { Thus } \phi=12.77 \text {. }
$$

$P_{2}^{\prime \prime \prime}=R_{x}(\phi) \cdot P_{2}^{\prime \prime}=R_{x}(\phi) \cdot R_{y}(\theta-90) \cdot P_{2}^{\prime}=R_{x}(\phi) \cdot R_{y}(\theta-90) \cdot T \cdot P_{2} \cdot 33$

$R_{x}(\phi)=\left[\begin{array}{cccc}1 & 0 & 0 & 0 \\ 0 & \cos \phi & -\sin \phi & 0 \\ 0 & \sin \phi & \cos \phi & 0 \\ 0 & 0 & 0 & 1\end{array}\right]=\left[\begin{array}{cccc}1 & 0 & 0 & 0 \\ 0 & 0.97 & -0.22 & 0 \\ 0 & 0.22 & 0.97 & 0 \\ 0 & 0 & 0 & 1\end{array}\right] .34$

So,

$$
\begin{aligned}
P_{2}^{\prime \prime \prime} & =\left[\begin{array}{cccc}
1 & 0 & 0 & 0 \\
0 & 0.97 & -0.22 & 0 \\
0 & 0.22 & 0.97 & 0 \\
0 & 0 & 0 & 1
\end{array}\right] \cdot\left[\begin{array}{cccc}
-0.60 & 0 & -0.97 & 0 \\
0 & 1 & 0 & 0 \\
0.79 & 0 & -0.60 & 0 \\
0 & 0 & 0 & 1
\end{array}\right] \cdot\left[\begin{array}{c}
106.12 \\
30.35 \\
-81.64 \\
1
\end{array}\right] \\
& =\left[\begin{array}{cccc}
1 & 0 & 0 & 0 \\
0 & 0.97 & -0.22 & 0 \\
0 & 0.22 & 0.97 & 0 \\
0 & 0 & 0 & 1
\end{array}\right] \cdot\left[\begin{array}{c}
0 \\
30.35 \\
133.89 \\
1
\end{array}\right]=\left[\begin{array}{c}
0 \\
0 \\
137.89 \\
1
\end{array}\right] \text { or }
\end{aligned}
$$

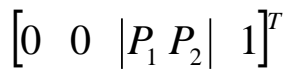
35

As can be seen, $P_{1}^{\prime \prime} P_{2}^{\prime \prime}$ is rotated into the Z-axis by $(\phi)$. The line segment $P_{1}^{\prime \prime} P_{3}^{\prime \prime}$ is not shown because it is not used to determine the angle of rotation.

\section{CONCLUSION}

A standard camera on a five degree-of-freedom fixture was used to feed video shots. A 3D transformation was used in order to calculate the absolute position and orientation of the camera. Good registration between the real and virtual scenes was achieved in the camera calibration. The transformation of the camcorder coordinates was used to set up the virtual sense, although minor dynamic errors were found.

\section{ACKNOWLEDGMENT}

The effort of the High Institute of Industrial TechnologyLibya is highly appreciated, for their unlimited support.

\section{REFERENCES}

[1] Alvarez, L, Henríquez, P., Mazorra, L., (2012), Mathematical Models for the Calibration of Cameras Mounted on a Tripod Using Primitive Tracking, 9th International Conference, ICIAR 2012, Aveiro, Portugal, Proceedings, Part I, Springer Berlin Heidelberg.(pp 304311).

[2] Bazargani , H. (2015) Camera calibration and pose estimation from planes, IEEE Instrumentation \& Measurement Magazine, Volume: 18, Issue: 6.

[3] Dias, P.(2003), Tsai Camera Calibration, Institute of Electronics and Informatics Engineering of Aveiro IEETA, Portugal.

[4] Foley J., Dam, A., Feiner, S., Hughes, J. and Phillips, R. (1994). Introduction to Computer Graphics, Wesley Publishing Company, Inc. USA 
[5] Roger T. (1987). A Versatile Camera Calibration Technique for HighAccuracy 3D Machine Vision Metrology Using Off-the-Shelf TV Cameras and Lenses," IEEE Journal of Robotics and Automation, Vol. RA-3, No. 4, (pp. 323-344).

[6] Sense8 Inc. (1999). NASA combines EAI and the Mathworks products to create a revolutionary space shuttle/space station docking simulation.

[7] Tsai, R., (2003) A versatile camera calibration technique for high-accuracy 3D machine vision metrology using off-the-shelf TV cameras and lenses, IEEE Journal on Robotics and Automation, USA, (pp. 323 - 344).

[8] Vasconcelos F., Barreto, J, Boyer, E. (2012) A Minimal Solution for Camera Calibration Using Independent Pairwise Correspondences, European Conference on Computer Vision, (pp.724-737). 